\title{
Wie publiziere ich einen Fallbericht in „Der Notarzt“?
}

\section{Zusammenfassung}

Fallberichte sind ein wichtiger Bestandteil medizinischer Fachliteratur. Der Notarzt veröffentlicht regelmäßig Case Reports aus dem Rettungsdienst und achtet dabei auf die Einhaltung von Publikationsstandards. Hierzu wird im Wesentlichen auf die 2013 erschienene CARE-Leitlinie (CARE: CAse REporting) zur Erstellung klinischer Fallberichte aufgebaut, die zum Ziel hat, Vollständigkeit und Transparenz von Fallberichten zu verbessern. Wie ein interessanter und hochwertiger Fallbericht aufgebaut wird und welche Voraussetzungen für die Publikation zu erfüllen sind, das wird in diesem Artikel dargestellt.

\section{„Warum schreibt man Fall- berichte?" - Hintergründe}

Die Medizin und insbesondere der Rettungsdienst sind voll von überraschenden und ungewöhnlichen Verläufen. Fallberichte in „Der Notarzt“ sind der Ort, an dem hierüber berichtet wird [1]. Fallberichte oder „case reports" gehören zur ältesten Form der medizinischen Schrift überhaupt. Bereits im Altertum berichteten Ärzte einander von ihren Beobachtungen und Therapieerkenntnissen und versuchten, dadurch das medizinische Wissen ihrer Zeit zu vergrößern. Fallberichte erweitern die medizinische Fachliteratur um Beobachtungen, die in klinischen Studien häufig nicht oder nur unvollständig berichtet werden können. Sie können die Entdeckung relevanter klinischer Probleme fördern und Hypothesen für zukünftige klinische Studien generieren. Fallberichte dienen somit medizinischen, wissenschaftlichen und zugleich andragogischen Zwecken. Daher haben gut verfasste Fallberichte immer auch einen wissenschaftlichen Wert, der nicht unterschätzt werden sollte [2]. Generell sollten Case Reports vor allem gut verständlich verfasst sein und sich nicht nur Experten inhaltlich erschließen.
Eine unzureichende Qualität von Case Reports ist leider regelmäßig zu beobachten und bedroht nicht zuletzt die Bedeutung dieser Schriftform. Insbesondere in der Notfallmedizin wurde bei insgesamt steigender Zahl der veröffentlichten Fallberichte von teils gravierenden Qualitätsmängeln berichtet. So zeigten mehr als die Hälfte der publizierten Case Reports aus 4 Peer-Review-Zeitschriften im Fach Notfallmedizin Mängel im Bereich der Vollständigkeit, Transparenz und Reproduzierbarkeit [3]. Deshalb wurden in den letzten Jahren Initiativen unternommen, um Qualitätssicherung nicht nur im Bereich der Originalarbeiten [4], sondern auch bei klinischen Fallberichten [2] durch konsensbasierte Leitlinien zu unterstützen.

Ein gut geschriebener Fallbericht umfasst Symptome und klinische Zeichen, die Diagnose und die Therapie sowie den weiteren Verlauf der Krankengeschichte des Patienten. Hierbei beschreiben Fallberichte ungewöhnliche oder bislang unbekannte Verläufe und ordnen den berichteten Fall anhand der aktuellen Literatur zum Thema ein. Sie können daher mit einem Case Report Ihren Kollegen Ihre Erfahrung weitergeben und die medizinische Leitung verbessern helfen. Nicht unerwähnt bleiben soll, dass der Thieme Verlag für Publikationen Autorenrabatte für Produkte des Verlages bietet.

\section{„Alle einverstanden?" - Konsens als Voraussetzung}

Ein Fallbericht gewährt immer auch einen Einblick in den Arbeitsbereich, in dem sich der Fall zugetragen hat. Dieser Einblick kann auch Schwächen oder Probleme aufzeigen. Nicht immer ist vorhersehbar, welche Nachfragen oder Kommentierungen ein Fallbericht hervorrufen wird. Daher gilt: Suchen Sie vor der Publikation einen breiten Konsens zur Veröffentlichung und stimmen Sie sich mit den Beteiligten und den Verantwortlichen (Vorgesetzten) ab. Vergessen Sie nicht, dass der Rettungs- dienst im Regelfall in der Trägerschaft eines Landkreises oder einer Stadt steht wer weiß, ob nicht auch ein politischer Mandatsträger auf Umwegen von der Veröffentlichung hört. Dasselbe gilt auch für die Schnittstellen zum Rettungsdienst: Vergessen Sie daher auch nicht, sich mit den weiterversorgenden Kollegen abzustimmen, welchen Inhalts Ihre Publikation sein wird. Bestenfalls erhalten Sie bei einer solchen Abstimmung wichtige Zusatzinformationen zu Ihrem Fall und gewinnen sogar einen kompetenten Koautor.

Eine Abstimmung im Vorfeld erspart nicht nur Ärger, sondern hilft Ihnen vielleicht auch in Zukunft, weitere Projekte in Ihrem Rettungsdienst durchzuführen und zu publizieren.

Nicht zuletzt: Versuchen Sie, wenn irgend möglich, auch den Patienten oder bei Verstorbenen seine Angehörigen zur Zustimmung zu einer Publikation zu bewegen. Aus ethischen Gründen wird inzwischen gefordert, bei jedem Fallbericht eine Zustimmung des Patienten einzuholen [2]. Die Zustimmung sollte schriftlich als „informed consent" erfolgen, der Patient also umfassend darüber informiert sein, was Inhalt der Veröffentlichung ist und dieser zustimmen. Kann der Patient keine Zustimmung geben und sind auch keine Angehörigen zu identifizieren, deren Zustimmung eingeholt werden könnte, so sollte die Entscheidung einer Ethikkommission eingeholt werden.

Vergessen Sie nicht: Kaum ein Rettungsdienst-Bereich ist so groß, dass nicht auch bei anonymer Berichterstattung die Möglichkeit besteht, dass jemand die Situation als die eigene Versorgung oder die eines Bekannten wiedererkennt. Spätestens bei spektakulären Unfällen ist dies regelhaft realistisch. Die Rechtsprechung zu Einsatz-Fotografien setzt zudem sehr enge Grenzen, wenn ein Einverständnis der Abgebildeten nicht vorliegt. Schon Tätowierungen oder auffälliger Körperschmuck könnten eine Identifizierung möglich ma- 
chen und sind daher ohne Zustimmung nicht verwendbar. $>$ Abb. 1 und $\mathbf{2}$ sind Beispiele für rechtlich einwandfreie Bebilderungen.

Kurz: Es spricht alles dafür, einen Fallbericht zuvor mit allen relevanten Persönlichkeiten abzustimmen und diese für eine Veröffentlichung zu gewinnen.

\section{„Wer ist dabei?“ - Autorenschaft}

Überlegen Sie sich gut, wen Sie an Ihrem Case Report beteiligen möchten. Wie schon beschrieben ist es oft sinnvoll, aus den verschiedenen an der Versorgung beteiligten Institutionen und Fachgebieten Koautoren zu gewinnen. Denken Sie auch an mögliche nichtmedizinische Koautoren, z. B. aus der Feuerwehr. Wenn Autoren aus verschiedenen Institutionen kommen, dann sollten diese auch genannt werden. Wer nicht an der Erstellung des Textes beteiligt war, der ist kein Autor Sie können aber Menschen, die am Einsatz beteiligt waren, in der Danksagung am Ende des Textes würdigen.

\section{„Und dann, und dann ..." - Aufbau eines Fallberichtes}

Schon der Titel sollte Wesentliches zu Ihrem Report transportieren: „Trecker-Unfall mit zunehmender Dyspnoe“, „Kindliche Bewusstseinsstörung nach versehentlicher Medikamenteneinnahme“. Auch das Wort „Fallbericht“ sollte im Titel enthalten sein, damit die Leser einen Bezug herstellen können.

Nach dem Titel werden von Ihnen Schlüsselwörter zum Fall definiert. Diese Schlüsselwörter machen Ihren Text später besser auffindbar, wenn z.B. in Datenbanken/ Suchsystemen nach relevanten Beiträgen gesucht wird.

Ein Fallbericht beginnt mit einer Zusammenfassung (engl. „Abstract“) der Ereignisse. Die Zusammenfassung soll einen Überblick geben und Interesse wecken. Es ist aber nicht nötig, in der Zusammenfassung schon „die Katze aus dem Sack“ zu lassen. Hat Ihr Fall hat eine überraschende

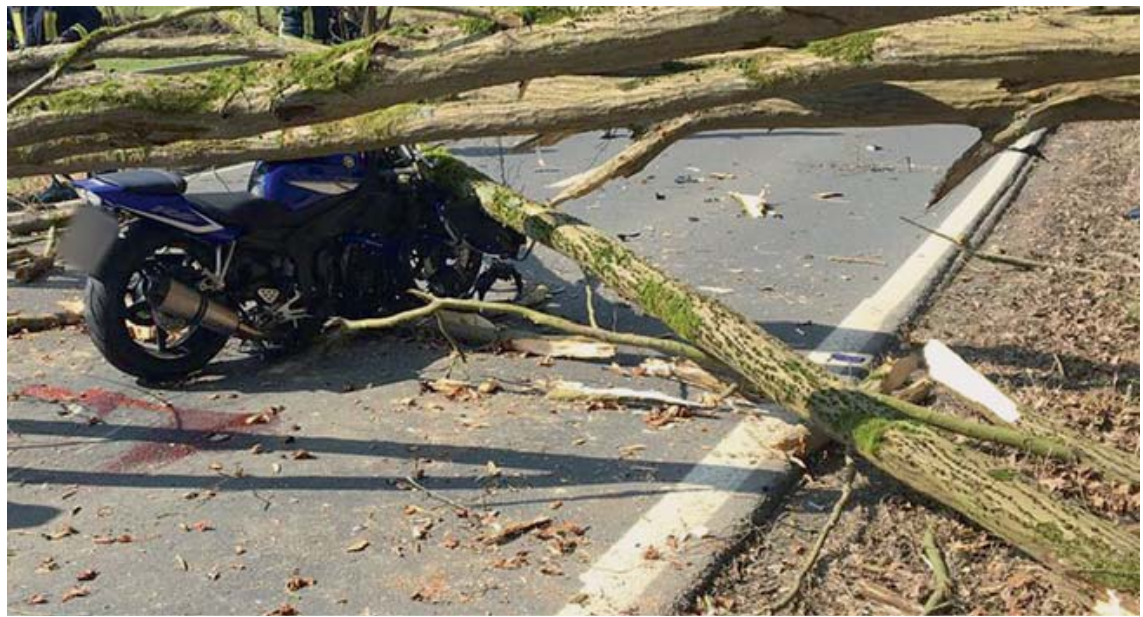

Abb. 1 Unfallstelle, keine Person erkennbar, keine personenbezogenen Daten enthalten.

Wendung, dann kündigen Sie lieber nur an, dass die Versorgung einen unerwarteten Verlauf genommen hat. Machen Sie Aussagen dazu, welche neuen Informationen der Fall liefert.

Der eigentliche Artikel beginnt mit einer Einleitung zum Thema - stimmen Sie damit die Leserschaft auf die Thematik ein, damit es besser gelingt, sich im nächsten Abschnitt auf den Fall an sich einzulassen.

Im Folgenden schildern Sie Ihren Fall. Vergessen Sie dabei nicht, die Auffindesituation und bedeutende Umgebungsbesonderheiten darzustellen. Was waren die Hauptbeschwerden des Patienten, welche Symptome wurden erkannt? Schildern Sie die erhobenen Befunde und die sich ergebenden (Verdachts-)Diagnosen. Stellen Sie die durchgeführten Interventionen dar und beschreiben Sie den weiteren Einsatzverlauf.

Stellen Sie den Patienten und seine wesentlichen demografischen Charakteristika dar und erwähnen Sie zumindest grob, welche relevanten Vorerkrankungen und Risiken bestanden.

Den Lesern hilft bei längeren Verläufen oft eine Zeitachse mit den wichtigsten Ereignissen. Hierbei reichen grobe Zeitangaben aus, helfen aber, den Ablauf besser nachvollziehen zu können. Vermeiden Sie es, die realen Uhrzeiten und das Datum des Einsatzes zu nennen, da dies die Anonymität der Beteiligten gefährdet.

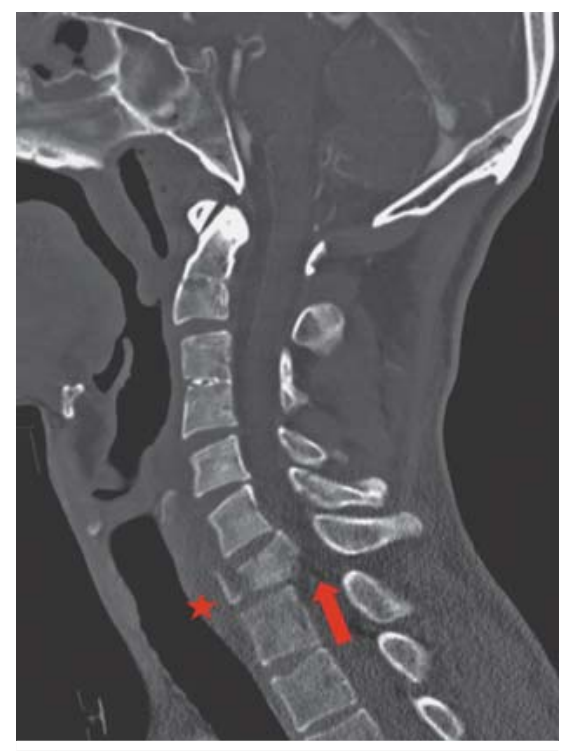

- Abb. 2 CT-Darstellung der HWS mit Trümmerfraktur des 7 . Halswirbels, keine Person erkennbar, keine personenbezogenen Daten enthalten.

Auch die Auswahl der Zielklinik, das gewählte Transportmittel und der Verlauf des Transportes sollten beschrieben werden. Nicht zuletzt: die Übergabe im Krankenhaus und der Zustand Ihres Patienten zu diesem Zeitpunkt. Stellen Sie dar, welche Diagnostik, Therapie, Interventionen erfolgten und welche Ergebnisse sich zeigten.

Wichtig: An dieser Stelle Ihres Textes ist noch nicht der Ort für Erklärungen und 
- Tab. 1 10-Punkte-Plan Case Report in „Der Notarzt“, nach Gagnier et al. [2].

\begin{tabular}{|c|c|c|}
\hline 1 & Titel & Erste Informationen werden vermittelt; enthält das Wort „Fallbericht“. \\
\hline 2 & Zusammenfassung (Abstract) & enthält in Kurzform: Einleitung, Fallbeschreibung, Schlussfolgerung \\
\hline 3 & Einleitung & Gibt einen Überblick und Hintergründe; enthält erste Hinweise zur medizinischen Literatur zum Fall. \\
\hline 4 & Patient & $\begin{array}{l}\text { Hier finden sich Angaben zu Demografie, Hauptsymptomen bei Einsatzbeginn, anamnestische Angaben, } \\
\text { Begleiterkrankungen. }\end{array}$ \\
\hline 5 & Befunde & $\begin{array}{l}\text { Die relevanten Befunde des Patienten werden dargestellt, ggf. entstandene Probleme bei der Befund- } \\
\text { interpretation werden aufgezeigt. }\end{array}$ \\
\hline 6 & Zeitachse/-strahl & In einer Tabelle oder einer Abbildung wird ein Eindruck von den Zeitabläufen vermittelt. \\
\hline 7 & Diagnostik & $\begin{array}{l}\text { Welche Diagnostik wurden eingesetzt und welche Überlegungen lagen der Entscheidung zugrunde? } \\
\text { Welche Resultate zeigten sich? Welche Herausforderungen bestanden? }\end{array}$ \\
\hline 8 & Interventionen & $\begin{array}{l}\text { Welche Verfahren wurden eingesetzt und welche Überlegungen lagen dem zugrunde? Gab es Alternativen? } \\
\text { Welche Ergebnisse zeigten sich? Welche Herausforderungen bestanden und gab es Komplikationen? }\end{array}$ \\
\hline 9 & Verlauf und Outcome & $\begin{array}{l}\text { Hier wird der Verlauf inkl. der Krankenhausdiagnostik und Therapie zusammengefasst. Wie ging es mit dem } \\
\text { Patienten weiter? Bei Verstorbenen werden idealerweise Ergebnisse der Obduktion dargestellt. }\end{array}$ \\
\hline 10 & Diskussion & $\begin{array}{l}\text { Diskutiert werden Stärken und Schwächen der Versorgung. Was hätte besser laufen können? Ideal ist } \\
\text { eine Erweiterung um die Patientenperspektive. Und: Was sagt die relevante medizinische Literatur zum } \\
\text { Thema? Was ist die Quintessenz des Falles? }\end{array}$ \\
\hline
\end{tabular}

Kommentierungen der Geschehnisse dies erfolgt erst in der Diskussion: beschreiben Sie, ohne zu bewerten.

Besonders interessant wird es für die Lesenden, wenn auch der weitere klinische Verlauf, bestenfalls bis zur Entlassung und der Rückkehr des Patienten in den Alltag, beschrieben werden kann. Ein Fallbericht, der an der Klinikpforte endet und keine Informationen über den weiteren Verlauf enthält, ist immer eine vertane Chance, Auswirkungen der Rettungsdienst-Versorgung auf das Gesamtergebnis darzustellen, und enttäuscht daher häufig die Leser.

Die Perspektive des Patienten und damit sein Erleben des geschilderten Falles und seiner Auswirkungen darzustellen, ist eine relativ neue Idee in der Gestaltung von Fallberichten [2], eröffnet für die Leserschaft aber neue und sehr spannende Einblicke. Versuchen Sie, wenn irgend möglich, auch die Perspektive des Patienten in Ihren Text aufzunehmen und in der Diskussion der Ereignisse zu berücksichtigen.

Spezielle Fragestellungen oder Techniken bilden Sie am besten außerhalb des Textes in einer sogenannten Infobox ab. Hierin können Sie einen erläuternden Sachverhalt darstellen, in dem der interessierte Leser ergänzende Informationen findet [1].
Am Ende der Falldarstellung folgt eine kurze Schlussbetrachtung, quasi die Quintessenz des Einsatzes aus Ihrer Sicht, welche die Überleitung in die Diskussion bietet.

\section{„Was lernen wir daraus?“ - die Diskussion im Fallbericht}

Die Diskussion ist das Herzstück Ihres Fallberichtes. Hier zeigt sich, was gut war und was hätte besser laufen können, und auch, ob sich Strukturen bewährt haben. Fester Bestandteil der Diskussion eines Fallberichtes ist eine kurze Darstellung der relevanten aktuellen Literatur zum Thema. Dies beinhaltet z. B. die Inzidenz der beschriebenen Erkrankung und deren Morbidität bzw. die Häufigkeit von Unfällen wie dem von Ihnen geschilderten sowie deren typische Verletzungsfolgen.

Ihre verwendete Literatur stellen Sie im Literaturverzeichnis am Ende des Textes zusammen, Informationen dazu finden Sie in den Autorenrichtlinien der Zeitschrift „Der Notarzt“.

Stellen Sie im Weiteren dar, was Besonderheiten Ihres Falles waren und welche Stärken und Schwächen die Versorgung des Rettungsdienstes hatte. Erklären Sie Umstände, die der Erklärung bedürfen, damit die Lesenden Ihr Vorgehen besser verstehen können.

\section{„Jetzt wird es ernst!“ - Einreichung des Fallberichtes}

Für die übersichtliche Orientierung haben wir einen „10-Punkte-Plan Case Report“ erstellt, der als Checkliste für die Erstellung eines veröffentlichungsfähigen Fallberichtes in „Der Notarzt“ dienen soll $(\vee$ Tab. 1).

Ferner für Autoren zu beachten: Verlagsvorgaben des Thieme Verlages [5]. Hier finden Sie Vorgaben zu Formaten, Umfang des Textes bis hin zum Finden korrekt gewählter „Tarnnamen“. Tabellen und Abbildungen müssen sich an den AutorenRichtlinien des Verlages orientieren, um eine problemlose Produktion zu ermöglichen. Auch Angaben zur Schreibweise zur Zitierweise und der Nennung von Interessenkonflikten finden Sie hier.

Das eigentliche Einreichen erfolgt über ein Onlinesystem des Verlages, alle Informationen hierzu finden Sie in der „Thieme Autorenlounge“. Ihr Fallbericht wird von den Schriftleitern der Zeitschrift und 2 unabhängigen Sachverständigen begutachtet. Über das Ergebnis werden Sie informiert. Nach Abschluss des Begutach- 
tungs-Prozesses erhalten Sie Korrekturabzüge, die Sie finalisieren und freigeben müssen. Danach ist die Druckfreigabe erfolgt und Sie halten schon wenig später das Belegexemplar Ihres Case Reports in den Händen - ein guter Moment!

\section{Lohnt sich das? - ein Plädoyer für den Fallbericht!}

Gut geschriebene Fallberichte sind eine Bereicherung für „Der Notarzt“ und für die notfallmedizinische Fachwelt. Der Aufwand für einen Fallbericht ist ohne Zweifel groß, umso wichtiger ist für Autoren wie Lesende der Nutzen des Textes. Ohne die Einhaltung von Standards haben Fallberichte nicht die nötige Schlüssigkeit, um Datenanalysen zu ermöglichen, wissenschaftliche Ansätze zu begründen und nicht zuletzt die klinische Praxis zu verbessern.
[1] Grohé M, Asmus J, Bohn A et al. Erkannt und konsequent erfolgreich behandelt: Reanimation bei Lungenarterienembolie. Notarzt 2020; 36: 27-32. doi:10.1055/a-1018-8971

[2] Gagnier JJ, Riley D, Altmann DG et al. The CARE guidelines: Consensus-based clinical case reporting guideline development. Dtsch Arzteb Int 2013; 110: 603-608. doi:10.3238/arztebl.2013.0603

[3] Richason TP, Paulson SM, Lowenstein SR et al. Case reports describing treatments in the emergency medical literature: missing and misleading information. BMC Emerg Med 2009; 9: 10

[4] Turner L, Shamseer L, Altmann DG et al. Does use of the CONSORT Statement impact the completeness of reporting of randomised controlled trials published in medical journals? A Cochrane review. Syst Rev 2012 (1): 60. doi:10.1186/2046-4053-1-60

[5] Georg Thieme Verlag KG. Thieme Autorenlounge (März 2020). Im Internet: https:// www.thieme.de/de/autorenlounge/93423. htm; Stand: 23.04.2020

\section{Autorinnen/Autoren}

\section{Andreas Bohn}

Prof. Dr., Stabsstelle Ärztliche Leitung Rettungsdienst, Berufsfeuerwehr Münster, Stadt Münster, Klinik für Anästhesiologie, operative Intensivmedizin und Schmerztherapie, Universitätsklinikum Münster

\section{Jens Asmus}

Dr., Stabsstelle Ärztliche Leitung Rettungsdienst, Berufsfeuerwehr Münster, Stadt Münster

\section{Korrespondenzadresse}

Prof. Dr. Andreas Bohn

Berufsfeuerwehr Münster York Ring 25

48159 Münster

BohnA@stadt-muenster.de 